\title{
EDITORIAL
}

\section{QUO VADIS EMERGENCY GENERAL SURGERY?}

\author{
V.M. Șurlin ${ }^{1,2}$, Ș. Pătrașcu ${ }^{1,2}$ \\ ${ }^{1} 1$ st Clinic of Surgery, Clinical County Emergency Hospital \\ ${ }^{2}$ University of Medicine and Pharmacy of Craiova
}

Emergency General Surgery (EGS) can be defined as surgery that is required to deal with an acute threat to life, or more specifically, to an organ, limb or tissue caused by external trauma, acute pathologic process, acute exacerbation of a chronic disease, or complication of a surgical or other interventional procedure [1]. In this type of surgery, the procedures are performed under the pressure of time, in order to preserve, restore and treat acute instabilities of the human system.

Nowadays, EGS is confronted with many different challenges both at a global and local level. Even in developed countries like the United States, in the last decade, there was an increase of $28 \%$ in hospital admissions that required EGS, consisting of a total of 27 million cases. The mortality risk for patients undergoing this type of surgery is 8 times higher than elective surgery. The financial projection for the next 40 years is showing a global burden of more than 41 billion dollars per year. Overall, emergency surgery is associated with $11 \%$ of hospital admissions and $50 \%$ of inhospital death [2].

In UK, there are 170000 emergency non-cardiac surgical interventions performed each year, of which 100.000 suffer complications. The crude rate of mortality was estimated at $14.7 \%$. As the population is aging, patients over 80 years old have a percentage of complications and mortality that reaches 50\% [3]. Chronic disorders such as cardiovascular, diabetes, and kidney disease will be more frequently encountered in advanced stages.

In Romanian medical teaching institutions such as Clinical County Emergency Hospitals, and Emergency Hospitals in Bucharest, which house residency programs, roughly 30-60\% of the surgical activity is estimated to be dedicated to EGS or acute care surgery. In low or middle developed countries the rate of EGS is more than $60 \%$ of all surgical interventions. 
There is a general tendency, also manifesting in our country, of less and less graduating medical students choosing general surgery as a specialization during residency. USA and Canada report a decreasing interest for general surgery from $12 \%$ in 1981 to 4-6\% in 2001 [4]. More than this, there is an attrition rate of general surgery residency programs worldwide and a similar situation can be found in Romania. In US and Canada a survey in 22 hospitals published in JAMA found a $25 \%$ attrition rate among women and $15 \%$ in men, for general surgery residents, most of them (48\%) leaving after the first year, for multiple reasons, mainly due to relocating to more attractive specialties or difficulties related to lifestyle [5]. In Romania, we witness the same phenomenon although there are no official statistics concerning this aspect. The number of female medical graduates has been constantly increasing along the years but few of them are willing to choose to specialize in general surgery.

A growing tendency for general surgeons is to overspecialize in a more restrictive field of surgery, addressing only one system or part of the digestive tract, like hepatobiliary, upper gastrointestinal, colo-rectal, breast, endocrine etc. In Europe, the percentage of overspecialization is estimated to be more than 50\% [4]. This is grounded on the old dictum stating that "practice makes better" and allows for a selection of cases. Breaking surgery in small pieces, however, will not help in situations like a blunt or penetrating abdominal trauma when multiple injuries of multiple organs are involved and should be handled through emergency surgery or damage control surgery. Indeed, those cases could also be managed by teams of specialized surgeons, but this is often not feasible and needs specific organization. In UK, a country that supported and introduced specialization in surgery, a joint document of the Association of Coloproctology of Great Britain and Ireland, the Association of Upper Gastrointestinal Surgeons, and the Association of Surgeons of Great Britain recognized the necessity for colorectal and upper gastrointestinal surgeons to be available alongside the EGS consultant in the emergency department, and to have prior exposure and training for emergency situations in their specific field [6].

More help for emergency surgery came from technology advances made in interventional angiology, radiology, and endoscopy. Nowadays, new procedures are replacing surgical interventions in some elective cases and are dealing with an important number of situations encountered in acute surgical patient (i.e. cholecystostomy for acute cholecystitis in severely ill patient, abscess drainage in diverticulitis or postoperative intraabdominal abscesses, sealing of 
bleeding vessels, endoscopic drainage of abscesses) and provide successful management of patient. Most of them are very well selected cases, but, nevertheless, there is still a plethora of complications that have to be managed by emergency surgery [7].

Minimally invasive surgery is constantly expanding and making its way in emergency cases (acute cholecystits, acute appendicits, diverticulitis, colonic obstruction, abdominal trauma, diagnostic laparoscopy), but requires specific expertise like in elective cases and back-up of open surgery in cases of conversion [5].

In US, UK and Europe, there is an increased awareness of the problem of unscheduled, emergency general surgery, generated by meticulous audits regarding the quality and the results of care. This led to the formation of working groups which established definitions, strategies for improvement and optimization of the organization, standards, recommendations, and guidelines for practice starting from analysis of the specific aspects of population, education, healthcare system, socio-economic, religious, and political peculiarities of every country $[1,2,6]$.

Twenty years ago, a model for Acute Care surgery was developed in US for trauma, surgical critical care and emergency general surgery. In the meantime, EGS fell behind in terms of guidelines and quality improvement. In this context, more attention and efforts should be put from now on in standardizing definitions, developing scales for severity assessment, national registries for open and non-operative management and standardized care [2].

In Europe, UEMS is considering emergency surgery as a "transferable competency" that needs knowledge of basic sciences, surgical anatomy, physiology, pathology, and clinical and technical skills [1].

The development of EGS needs specific formation along with necessary recognition and outcomes evaluation and performance improvement [8].

The European Society for Trauma and Emergency Surgery was formed 2007 by merging the European Association for Trauma \& Emergency Surgery and the European Trauma Society. According to its mission statement, since then, it has continued to promote best practice in the provision of emergency and trauma surgery from pre-hospital care through diagnosis, intervention, and intensive care to rehabilitation. This is underpinned by international collaboration, scientific research, development and delivery of training courses, and the work of the specialist sections (Disaster \& Military Surgery, Emergency Surgery, Polytrauma, Visceral Trauma as well as Skeletal Trauma and Sports Medicine). 
Romanian Society for Trauma and Emergency Surgery (RSTES) was formed in 2005. In 2017, the European Congress of Trauma and Emergency Surgery took place in Bucharest. Many international workshops on subjects related to emergency surgery were organized [7]. Every edition of the National Congresses of the Romanian Society of Surgery and National Conferences included sessions debating subjects of emergency surgery, involving local and international speakers.

Emergency surgery exposes the surgeon to a miriad of situations, therefore a division of the surgical practice in small parts would confront the case of the patient with a "puzzled surgical team" that will try to fit and cure, situations that are more easily predicted in elective surgery [9].

Obviously nowadays it is becoming increasingly difficult to attract young people to general surgery, to make them perform emergency surgery, and to keep them motivated along the years while over specialization and private practice is much more attractive. Nevertheless, even private general hospitals have to deal with emergencies from either the community, as patients are seeking it directly, or from complication of their own surgical interventions [10].

As much as we need experts in every subspecialization of surgery, as this strategy demonstrated increased performance, we also need experts, teachers and leaders in emergency surgery in order to treat the patients, to teach the younger generations and, equally important, to publish and share their results [11].

In the end, even if the mighty queen called "General Surgery" gave birth to many princess such as hepatobiliary, upper GI, lower GI, or endocrine surgery, EGS or acute care surgery, as we may like to call it, is its rightful sister and should no longer be considered like a Cinderella, but an equal in all terms, because she is going to be around for many years to come and should be treated with the same level of appreciation, consideration and respect.

\section{References}

[1]https://www.uemssurg.org/divisions/emergency-surgery

[2]Havens JM, Neimann PU, Campbell PL, Croce AM, Spain AD, Napolitano AM, The Future of Geneal Emergency Surgery, Ann Surg 2019;270:221-222

[3]https://www.rcseng.ac.uk/-/media/files/rcs/aboutrcs/regional/rcs_emergency_surgery_2011/wed.pdf 
[4]Felice GA, General surgery - is the general dead?, www.uems.eu>_data>assets>pdf.file>Felice-A.-General-Surgery.-Is-the-General-Dead

[5]Yeo HL, Abelson JS, Symer MM, et. al. Association of Time to Attrition in Surgical Residency With Individual Resident and Programmatic Factors. JAMA Surg. 2018.

[6]Anderson I, Future of Emergency Surgery - Joint document of the Association of Coloproctology of Great Britain and Ireland, Association of Upper Gastrointestinal Surgeons, Association of Surgeons of Great Britain and Ireland https://www.acpgbi.org.uk/content/uploads/2016/07/Future-of-EGS-joint-document_IainAnderson_140915.pdf

[7]Beuran M, Diaconescu B, Emergency Surgery in Romania - Present and Perspectives, Chirurgia, 2017, 112(5):503-504

[8]Coccolini F, Kluger Y, Ansaloni L, Moore EE, Coimbra R, Fraga GP, Kirkpatrick A, Peitzman A, Maier R, Baiocchi G, Agnoletti V, Gamberini E, Lepanniemi A, Ivatury R, Sugrue M, Sartelli M, Di Saverio S, Biffl W, Catena F, WSES worldwide emergency general surgery formation and evaluation project, World J Emergency Surgery 2018, 13:13

[9]Catena F, Moore EE. World Journal of Emergency Surgery (WJES), World Society of Emergency Surgery (WSES) and the role of emergency surgery in the world. World J Emerg Surg 2007;2,3

[10]To KB, Kamdar NS, Patil P, Collins SD, Seese E, Krapohl DL, Campbell Jr DS, Hemilla MR, Englesbe MJ, Napolitano ML Acute Care Surgery Model and Outcomes in Emergency General Surgery for the Michigan Surgical Quality Collaborative (MSQC) Emergency General Surgery Study Group and the MSQC Research Advisory Group $J$ Am Coll Surg. 2019;228(1):21-28.e7.

[11]Mullen MG, Michaels AD, Mehaffey H, Guidry AC, Turrentine FE, Hedrik TL, Friel CM, Risk Associated With Complications and Mortality After Urgent Surgery vs Elective and Emergency Surgery Implications for Defining "Quality" and Reporting Outcomes for Urgent Surgery, JAMA Surg. 2017;152(8):768-774. 